\title{
Near-field scanning optical microscopy of photonic crystal high-Q nanocavities
}

\author{
Koichi Okamoto, Marko Lončar, Tomoyuki Yoshie and Axel Scherer: \\ Department of Electrical Engineering, California Institute of Technology, Pasadena, CA 91125-9300 \\ Yueming Qiu, Pawan Gogna \\ In Situ Technology and Experiments System Section, Jet Propulsion Laboratory, California Institute of Technology, \\ MS 302-306, 4800 OakGrove Drive, Pasadena, CA 91109
}

\begin{abstract}
Near-field scanning optical microscopy (NSOM) was used to observe high-resolution images of planar photonic crystal nanocavities fabricated in active InGaAsP material. We have observed the smallest optical cavity modes and dielectric band modes.

(C2000 Optical Society of America

OCIS codes: $(000.0000)$ General
\end{abstract}

Recently, near-field scanning optical microscopy (NSOM) has been used as a powerful alternative method to analyze local electromagnetic field distributions on fabricated nanophotonic structures. Several groups have reported NSOM studies of planar photonic crystals (PPC) [1-2], however, high spatially resolved near-field images of modes inside the PPC cavities have not so far been reported. We report optical mode images obtained by NSOM on compact PPC cavities based on fractional edge dislocations [3-4].

A cw light from a He-Ne laser $(633 \mathrm{~nm})$ and a $20 \mathrm{~ns}$ pulsed diode laser $(780 \mathrm{~nm})$ were used as the excitation laser source for illumination mode (I-mode) and the collection mode (C-mode) measurements, respectively. Photoluminescence (PL) signals were filtered out from the excitation laser by a low-pass filter with a cut-off wavelength of $850 \mathrm{~nm}$, and detected with a high-sensitivity (fW) InGaAs photo-detector. A metal-coated fiber tip, with small aperture size, enables us to substantially cut leaky mode light. The best resolution in our system is as small as $50 \mathrm{~nm}$. By using the shear-force detection, we could also obtain a topographic image of the sample, in addition to the near-field optical image. We have also performed micro-PL measurements [3-5] on our structures in order to confirm the existence of the localized cavity modes. The photonic crystal nanocavities fabricated in active InGaAsP material used in this work are very similar to those used to realize low-threshold lasers described in our previous publication [5].
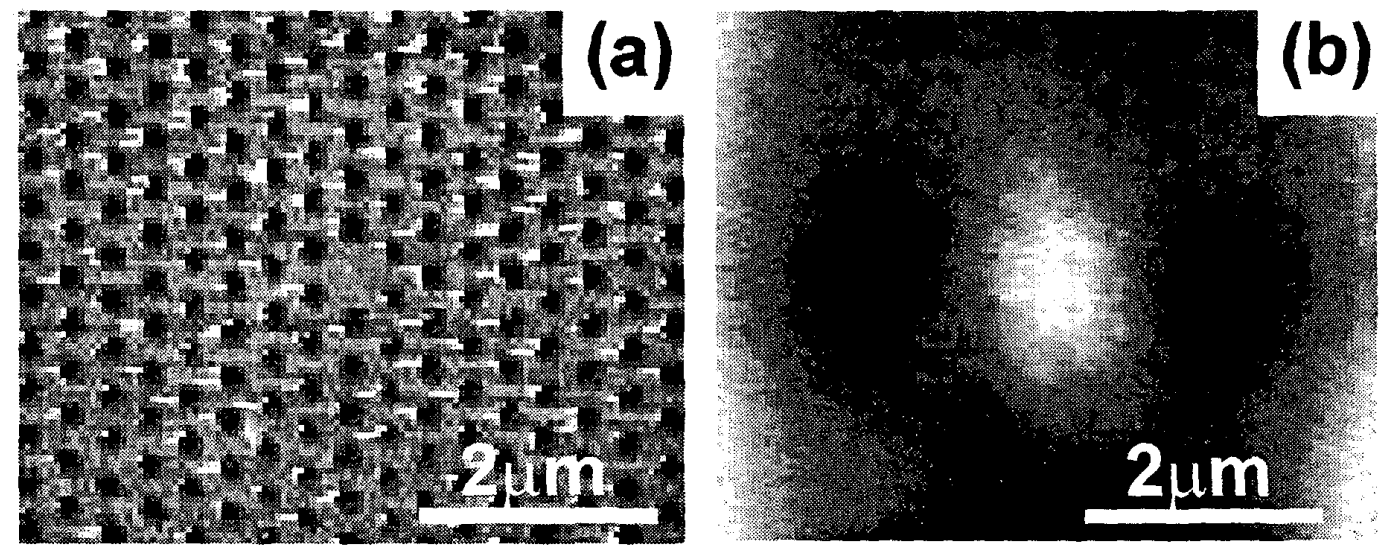

Fig. 1. (a) Topographic image obtained by the shear-force microscopy. (b) Near-field photoluminescence (PL) image. 
Fig. 1 (a) and (b) show the topographic image and the near-field PL image of the photonic crystal nano-cavity. The bright spot seen in NSOM-PL image is located at the center of the PC structure, matching the position of the defect cavity, as shown in topographic image. This small spot size is an indication of a small mode volume, as expected from the localized cavity modes. To the best of our knowledge, the optical modes and field distribution that we have observed are the smallest reported so far. We also observed the micro-PL analysis of this structure. Two peaks positioned around $\lambda=1450 \mathrm{~nm}$ correspond to the localized dipole modes

In addition to localized cavity modes, we have observed dielectric band modes in bulk photonic crystals surrounding the nanocavity with $\mathrm{C}$-mode by geometrical tuning for eliminating localized modes out of the emission range. Similar results were predicted by theory [6], and profiles of dielectric band modes have been found using FDTD analysis of NSOM experiments. Also, spectra obtained using micro PL show the presence of dielectric bands at $\lambda=1555 \mathrm{~nm}$. We conclude that near-field scanning optical microscopy is a powerful tool for investigation of local profiles of confined modes in nanocavities.

[1] D. Gérard, L. Berguiga, F. de Fornel, L. Salomon, C. Seassal, X. Letartre, P. Viktorovitch, "Near-field probing of active photonic-crystal structures" Opt. Lett. 27, $173(2002)$.

[2] D.-J. Shin, S.-H. Kim, J.-K. Hwang, H.-Y. Ryu, H.-G. Park, D.-S. Song, Y.-H. Lee, "Far- and Near-Field Investigations on the Lasing Modes in Two-Dimensional Photonic Crystal Slab Lasers" IEEE J. Quantum Electronics, 38, 857 (2002).

[3] J. Vučković, M. Lončar, H. Mabuchi, A. Scherer, "Design of photonic crystal microcavities for cavity QED" Phys. Rev. E. 65, 16608 (2001). [4] T. Yoshie, J. Vučković, A. Scherer, H. Chen, D. Deppe, "Hligh qualtiy two dimensional photonic crystal slab cavities" Appl. Phys. Lett. 79, 4289 (2001).

[5] M. Lončar, T. Yoshie, A. Scherer, P. Gogna and Y. Qiu, "Low-threshold photonic crystal laser" Appl. Phys. Lett. 81, 2680 (2002)

[6] S. Fan, A. Ian, J. D. Joannopoulos, "Near-field scanning optical microscopy as a simultaneous probe of fields and band structure of photonic crystals: A computational study" Appl. Phys. Lett. 75, 3461 (1999). 